\title{
Cancer Incidence 2010-2014 Among the North Cyprus Population of Adults Aged 15 and Over
}

\author{
Nuriye SANCAR, ${ }^{1}$ Meltem NALÇA ANDRIEU,, ${ }^{2}$ Evren HINÇAL, ${ }^{1}$ Deniz GRANiT \\ 'Department of Mathematics, Near East University Faculty of Arts and Sciences, Nicosia-North Cyprus \\ ${ }^{2}$ Department of Radiation Oncology, Near East University Faculty of Medicine, Nicosia-North Cyprus \\ ${ }^{3}$ Department of Internal Medicine, Near East University Faculty of Medicine, Nicosia-North Cyprus
}

\section{OBJECTIVE}

This study is an analysis of cancer incidence among people aged 15 and over in North Cyprus (NC) with aim of helping to establish a cancer control plan based on the results.

\section{METHODS}

Data from 2010 to 2014 were obtained from Near East University (NEU) Hospital. Crude incidence rate (CIR) and age-standardized rate (ASR) per 100,000 were calculated for 28 different cancers for males and females. In line with GLOBOCAN 2012, ASR values for 2012 in NC were compared with South Cyprus (SC) and Southern Europe (SE).

\section{RESULTS}

Total of 1,782 cancer cases were diagnosed. ASR value was 180.61 and 192.75 per 100,000 person-years for males and females, respectively. Most prevalent male (M) cancers were lung, prostate, colorectal, thyroid, and bladder. Similarly, for females (F), they were breast, thyroid, colorectal, lung, and non-melanoma skin cancer. Thyroid cancer in females in NC was more prevalent than in SE. Breast (F), prostate, colorectal (F), bladder (M), and ovary and corpus were less frequent than in SE and SC; cervix, larynx (M), brain (M) and colorectal (M) only were less frequent than in SE, and the remainder were statistically similar.

\section{CONCLUSION}

Possible risk factors for the most common cancers in NC have been discussed; however, etiopathogenetic scientific results are still needed. Risk factor studies should be performed to raise public awareness and to plan future cancer prevention measures. The development of active cancer registry centers and combining data under a single cancer organization is recommended.

Keywords: Adult; cancer incidence; comparison; North Cyprus.

Copyright $\odot$ 2017, Turkish Society for Radiation Oncology

\section{Introduction}

Cancer is a major health problem in many developed and developing countries, where it is often the second most common cause of death for all age groups.[1,2] The purpose of the present study was to analyze information on cancer incidence among people aged 15 or over in North Cyprus (NC), which has a population of approximately 212,740 . This study will be beneficial to determine the forms of cancer that are more common, which will make it easier to combat the modifiable factors associated with them. Apart from the fact that this is the second study of its kind in NC, the country is deemed potentially interesting from an epidemiologi- 
cal perspective. Cancer is one of the leading causes of death throughout the world, demonstrated by the fact that in 2012, there was a total of 8.2 million cancer deaths. [3] The five main cancers that result in death can be grouped into: stomach, lung, liver, colorectal, and breast cancers. [3] However, 30\% of cancer deaths could be prevented by reducing the changeable risk factors such as being overweight or obese, tobacco use, unhealthy nutrition with minimal consumption of fruits and vegetables, alcohol consumption and lack of physical activity.[4] It is expected that annual cancer cases will increase from 14 million in 2012 to 22 million within the next two decades.[3] Furthermore, it is possible that the inter-communal strife and fighting that NC has experienced over the last 40 years has had adverse effects on cancer incidence in the country.[5,6]

As part of this study, we have reviewed the cancer incidence information available for the period between 2010-2014. The study presents the findings of the NEU Hospital Radiation Oncology and Medical Oncology Departments. The NEU Hospital Oncology Departments are the one of the main sources of cancer information on the island and codes the sites, types and morphological characteristics of cancer cases using the International Classification of Diseases for Oncology.[7]

For the purposes of the present analyses, only the registered cancer cases that were first diagnosed between $1^{\text {st }}$ January 2010 and $31^{\text {st }}$ December 2014 were considered. Tourists and persons who had not been resident in NC for more than six months prior to their cancer diagnosis were not included in the registry. All information received was treated as "confidential" and was approved by the ethical committee of the university.

\section{Materials and Methods}

Patient data was analyzed from the records of all departments of NEU Hospital from the period 20102014, but primarily from the Oncology Department. The files were examined for cancer patients aged over 15 in order to determine the ID code of the patient as well as their name (in confidence), age, gender, nature of cancer, date and the place of diagnosis. The registered cases were re-examined using Microsoft Excel in order to eliminate possible repeated registrations, duplicates or multiple diagnosis for the same patients so that metastatic cases were removed. Tourists and persons who had not been resident in NC for more than six months prior to their cancer diagnosis were not included in the registry. Primary cancer sites (topography) were coded according to the International
Classification of Disease (ICD, $10^{\text {th }}$ revision) codes.[7] The following cancer groups were analyzed: head-neck cancers (lip, oral cavity (C00-08), nasopharynx (C11), other pharynx (C09-10, C12-14) and larynx (C32)), upper gastrointestinal cancers (Esophagus (C15), stomach (C16), liver and intrahepatic bile ducts (C22), gallbladder and extrahepatic ducts (C23-24) and pancreas (C25)), colorectal cancers (C18-21), melanoma of skin (C43), non-melanoma skin (C44), trachea, bronchus and lung (C33-34), breast (C50), gynaecological cancers (cervix uteri(C53), corpus uteri (C54), ovary (C56) and other female genital cancers (C57)), brainnervous system cancers (C71-72), prostate (C61), testicular (C62), bladder (C67), kidney, renal pelvis and ureter (C64-C66), hematologic cancers (Hodgkin lymphoma (C81), non-Hodgkin lymphoma(NHL) (C82-85,C96), multiple myeloma and immunoproliferative diseases (C88, C90), leukaemia (C91-95) and thyroid(C73). Male and female cases were examined and analyzed separately.

As NEU Hospital does not have a pediatric oncology clinic, our research does therefore not include patients under 15 years old. According to the census in 2011, the North Cyprus population was 212,740, including $100,728(47.35 \%)$ women and 112,012 (52.65\%) men in the 15 and over age group.[8] The Crude Incidence Rates (CIRs) and Age Standardized Rates (ASRs) were calculated per 100,000 population. The comparison of Crude Incidence Rates can often be interpreted incorrectly because of differences in the age structure of the populations to be compared. This can be prevented by age-standardization. [9] In or study, the ASR (Age Standardized Rate) was calculated through the direct standardization method by using Microsoft Excel, based on the World Standard Population modified by Doll et al., in 1966.[10] 95\% Confidence Intervals (CIs) were constructed for ASR per 100,000 population for the 15 and over age group (Table 1). The ASR was computed for the most prevalent cancers for males and females in different age groups (Table 2). In line wth the Globocan 2012 research results,[11] the ASR values for 2012 were compared separately for males and females above the age of 15 in South Cyprus (SC) and the Countries of Southern Europe (SE). As the p-value was less than 0.001 , it was verified as statistically significant. We used the following formula for this analysis:

$$
\begin{aligned}
& \left(A S R_{1} / A S R_{2}\right)^{1 \pm\left(Z_{\alpha / 2} / x\right)} \\
& x=\frac{A S R_{1}-A S R_{2}}{\sqrt{\left(s . e .\left(A S R_{1}\right)\right)^{2}+\left(s . e .\left(A S R_{2}\right)\right)^{2}}}, Z_{\alpha / 2}=3.29, \text { s.e. (ASR): }
\end{aligned}
$$

standard error of ASR. 
Table 1 Crude Incidence Rate (CIR) and Age Standardized Rate (ASR) of cancers, per 100,000 population aged 15 and over, according to cancer site and gender in North Cyprus; 2010-2014

\begin{tabular}{|c|c|c|c|c|c|c|c|c|}
\hline \multirow[t]{2}{*}{ Topography (C-code) } & \multicolumn{4}{|c|}{ Male } & \multicolumn{4}{|c|}{ Female } \\
\hline & $\mathbf{n}$ & $\%$ & CIR & ASR (CI 95\%) & $\mathbf{n}$ & $\%$ & CIR & ASR (Cl 95\%) \\
\hline Head and neck cancers & 65 & 7.7 & 11.61 & $14.58(11.83-17.34)$ & 18 & 1.9 & 3.57 & $3.56(2.27-4.86)$ \\
\hline Lip-oral cavity (C00-08) & 22 & $2.6\left(33.8^{*}\right)$ & 3.93 & $4.99(3.37-6.61)$ & 11 & $1.2\left(61.1^{*}\right)$ & 2.18 & $2.21(1.18-3.24)$ \\
\hline Nasopharynx (C11) & 7 & $0.8(10.8)$ & 1.25 & $1.49(0.63-2.34)$ & 4 & $0.4(22.2)$ & 0.79 & $0.82(0.19-1.45)$ \\
\hline \multicolumn{9}{|l|}{ Other pharynx } \\
\hline (C09-10, C12-14) & 6 & $0.7(9.2)$ & 1.07 & $1.28(0.48-2.08)$ & 3 & $0.3(16.7)$ & 0.60 & $0.53(0.06-0.99)$ \\
\hline Larynx (C32) & 30 & $3.6(46.2)$ & 5.36 & $6.83(4.92-8.73)$ & - & - & - & - \\
\hline Upper gastrointestinal cancers & 72 & 8.6 & 12.86 & $15.95(13.09-18.81)$ & 57 & 6.0 & 11.32 & $11.64(9.26-14.01)$ \\
\hline Esophagus (C15) & 6 & $0.7\left(8.3^{* *}\right)$ & 1.07 & $1.31(0.49-2.12)$ & 5 & $1.7\left(8.8^{* *}\right)$ & 0.99 & $1.08(0.34-1.82)$ \\
\hline Stomach (C16) & 27 & $3.3(37.5)$ & 4.82 & $5.94(4.20-7.68)$ & 24 & $2.4(42.1)$ & 4.77 & $4.84(3.31-6.37)$ \\
\hline Liver (C22) & 17 & $2.0(23.6)$ & 3.04 & $3.79(2.39-5.20)$ & 10 & $1.1(17.5)$ & 1.99 & $1.93(0.98-2.87)$ \\
\hline Gallbladder (C23-24) & 5 & $0.6(6.9)$ & 0.89 & $1.19(0.38-2.00)$ & 7 & $0.6(12.3)$ & 1.39 & $1.43(0.60-2.26)$ \\
\hline Pancreas (C25) & 17 & $2.0(23.6)$ & 3.04 & $3.72(2.34-5.09)$ & 11 & $1.2(19.3)$ & 2.18 & $2.36(1.27-3.45)$ \\
\hline Colorectal (C18-21) & 91 & 10.8 & 16.25 & $20.38(17.13-23.63)$ & 48 & 5.1 & 9.53 & $9.57(7.44-11.7)$ \\
\hline \multicolumn{9}{|l|}{ Trachea, bronchus and lung } \\
\hline$(\mathrm{C} 33-34)$ & 169 & 20.1 & 30.18 & $37.16(32.83-41.49)$ & 41 & 4.3 & 8.14 & $8.49(6.45-10.52)$ \\
\hline Melanoma of skin (C43) & 14 & 1.7 & 2.49 & $2.99(1.77-4.22)$ & 23 & 2.4 & 4.57 & $4.26(2.90-5.63)$ \\
\hline Non melanoma skin (C44) & 33 & 3.9 & 5.89 & $6.97(5.12-8.81)$ & 37 & 3.9 & 7.35 & $7.24(5.4-9.07)$ \\
\hline Breast (C50) & 12 & 1.4 & 2.14 & $2.64(1.48-3.81)$ & 340 & 36.1 & 67.51 & 71.31 (65.4-77.21) \\
\hline Gynaecological cancers & - & - & N/A & - & 88 & 9.3 & 17.47 & 17.83 (14.9-20.76) \\
\hline Cervix uteri (C53) & _- & - & N/A & _- & 24 & $2.5\left(27.2^{* * *}\right)$ & 4.77 & $5.09(3.5-6.69)$ \\
\hline Corpus uteri (C54) & - & _- & $\mathrm{N} / \mathrm{A}$ & _- & 29 & $3.1(33.0)$ & 5.76 & $5.26(3.75-6.77)$ \\
\hline Ovary (C56) & _ & _- & N/A & _- & 27 & $2.9(30.7)$ & 5.36 & $5.85(4.12-7.57)$ \\
\hline Other gynaecological & & & & & & & & \\
\hline cancers (C57) & - & - & $\mathrm{N} / \mathrm{A}$ & - & 8 & $0.8(9.1)$ & 1.59 & $1.63(0.74-2.51)$ \\
\hline Prostate (C61) & 130 & 15.5 & 23.21 & 28.55 (24.75-32.34) & - & - & N/A & - \\
\hline Testicular (C62) & 19 & 2.3 & 3.39 & $3.25(2.1-4.39)$ & _- & _- & N/A & _- \\
\hline \multicolumn{9}{|l|}{ Kidney, renal pelvis, ureter } \\
\hline$(\mathrm{C} 64-66)$ & 12 & 1.4 & 2.14 & $2.48(1.38-3.59)$ & 2 & 0.2 & 0.39 & $0.45(-0.03-0.94)$ \\
\hline Bladder (C67) & 40 & 4.8 & 7.14 & $8.83(6.7-10.96)$ & 14 & 1.5 & 2.78 & $2.69(1.58-3.81)$ \\
\hline Brain, nervous system (C71-72) & 31 & 3.7 & 5.54 & $6.10(4.41-7.78)$ & 34 & 3.6 & 6.75 & $6.64(4.89-8.38)$ \\
\hline Thyroid (C73) & 52 & 6.2 & 9.28 & $10.10(7.94-12.26)$ & 160 & 17.0 & 31.77 & $33.12(29.11-37.12)$ \\
\hline Hematological cancers & 70 & 8.3 & 12.50 & $14.51(11.85-17.17)$ & 66 & 7.0 & 13.10 & $13.18(10.68-15.67)$ \\
\hline Hodgkin lymphoma (C81) & 13 & $1.5\left(18.6^{* * * *}\right)$ & 2.32 & $2.39(1.37-3.41)$ & 15 & $1.6\left(22.7^{* * * *}\right)$ & 2.98 & $3.06(1.85-4.27)$ \\
\hline \multicolumn{9}{|l|}{ Non-Hodgkin lymphoma } \\
\hline$(\mathrm{C} 82-85, \mathrm{C} 96)$ & 38 & $4.5(54.3)$ & 6.78 & 8.07 (6.06-10.08) & 35 & $3.7(53.0)$ & 6.95 & $6.96(5.15-8.77)$ \\
\hline Multiple myeloma (C90) & 15 & $1.8(21.4)$ & 2.68 & $3.18(1.93-4.43)$ & 11 & $1.2(16.7)$ & 2.18 & $2.17(1.16-3.18)$ \\
\hline Leukaemia (C91-95) & 4 & $0.5(5.7)$ & 0.71 & $0.87(0.21-1.52)$ & 5 & $0.5(7.6)$ & 0.79 & $0.78(0.18-1.38)$ \\
\hline \multicolumn{9}{|l|}{ Unknown Primary sites } \\
\hline$(\mathrm{C} 80, \mathrm{C76})$ & 29 & 3.5 & 5.17 & $6.16(4.14-7.91)$ & 15 & 1.6 & 2.97 & $2.78(1.67-3.89)$ \\
\hline All cancers & 839 & 100 & 149.81 & 180.67 (171.4-189.98) & 943 & 100 & 187.24 & $192.75(183.2-202.3)$ \\
\hline
\end{tabular}

* Within head - neck cancers; ${ }^{* *}$ within upper gastrointestinal cancers; ${ }^{* * *}$ within gynaecological cancers; ${ }^{* * * *}$ within hematological cancers.

If the interval does not include 1 , the standardized rates $A S R_{1}$ and $A S R_{2}$ are significantly different $(\mathrm{p}<0.001)$.

The confidence intervals were calculated for male and female patientsages and the two-tailed t-test was used with the aim of determining whether there was a statistically significant age difference between males and females. The Chi-Square test was used to define the differences between male and female patients in relation to the percentages of cancer sites. Further confirmatory analysis was performed using SPSS (version 20), (SPSS for Windows, Chicago, IL, USA). 


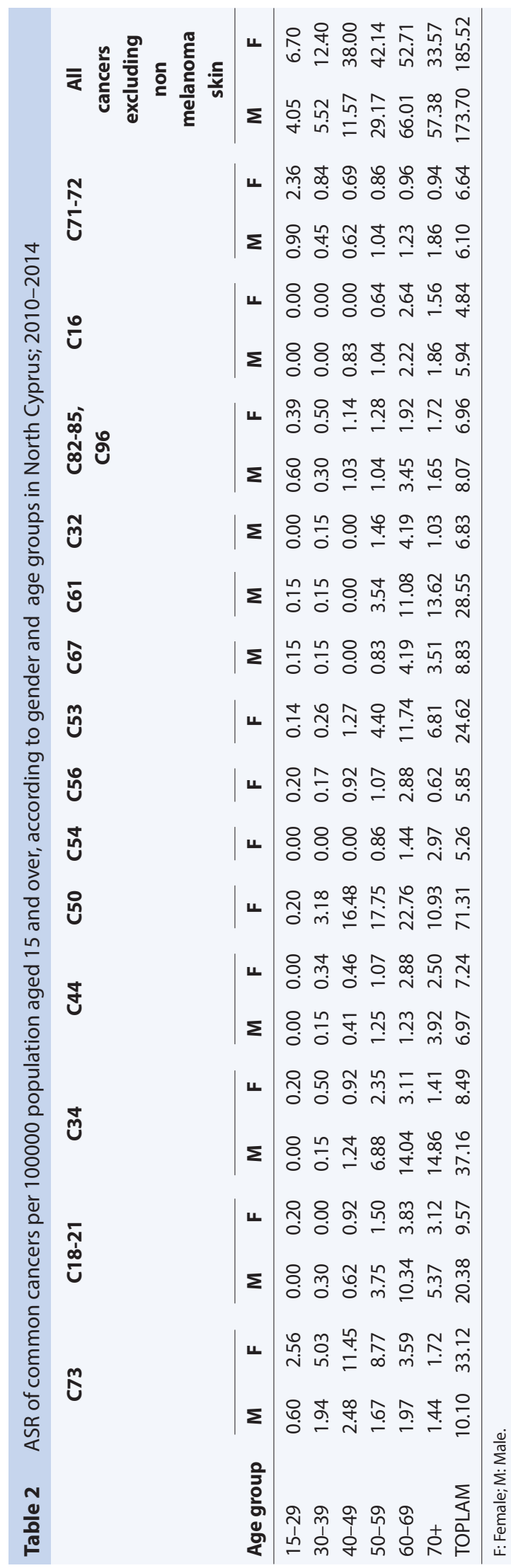

\section{Results}

A total number of 1,782 cancer cases were diagnosed among people aged 15 or over during the period 20102014 at NEU Hospital in North Cyprus. There were 839 male (47.1\%) and 943 (52.9\%) female cancer patients with a gender ratio of 1.12 (112 female patients for every 100 male patients). The average age of the patients was 60.6 years (CI 95\%: 59.9-61.3) with 58.1 years (CI 95\%: 57.2-59.1) for women and 63.5 (CI 95\%: 62.5$64.5)$ for men. The average age difference between men and women was statistically different $(\mathrm{p}<0.001)$.

From 2010 to 2014, the most common cancer sites were female breast (340 cases, $19.4 \%$ of all cancer cases), thyroid ( 212 cases, $11.9 \%$ ), lung ( 210 cases, $11.8 \%$ ), colorectal (139 cases, $7.8 \%)$ and prostate (130 cases, $7.3 \%)$. Lung (169 cases, $20.1 \%$ of all cancer cases in male patients), prostate (130 cases, $15.5 \%)$, colorectal (91 cases, $10.8 \%$ ), thyroid (52 cases, $6.2 \%$ ), bladder (40 cases, $4.8 \%$ ), non-Hodgkin lymphoma (38 cases, $4.5 \%$ ) and non-melanoma skin (33 cases, 3.9\%) cancers were the most prevalent cancers in male patients. For female patients, the most prevalent cancers were breast $(340$ cases, $36.1 \%$ of all cancer cases in female patients), thyroid (160 cases, 17.0\%), colorectal (48 cases, 5.1\%), lung (41 cases, $4.3 \%$ ), non-melanoma skin (37 cases, $3.9 \%$ ), non-Hodgkin lymphoma (35 cases, 3.7\%) and brain-nervous system $(34,3.6 \%)$, respectively. During this five year period, the standardized incidence rates of lung, colorectal, kidney-renal pelvis ureter and bladder cancers were significantly higher in men than in women among the population aged 15 and over. Breast and thyroid cancers were significantly higher for women $(\mathrm{p}<0.001)$ (Table 1).

When males were compared based on age range for the most common types of cancer, lung and prostate had the highest incidence rates in the 70 and over age group. Colorectal and bladder cancers had the highest incidence between the ages of 60 and 69. When females were compared based on age range for the most common sites of cancer, breast, colorectal, lung and non-melanoma skin had the highest incidence rates between the ages of 60 and 69 years. Most cancers had the highest incidence rates among the age groups 60-69 and 70+. In the younger age group, testicular cancer had the highest incidence rate. In females, thyroid cancer and malignant brain tumors had the highest incidence rates in the middle age group. For both genders combined, lung and colorectal cancers had the highest incidence between the ages of 60 and 69 years (Fig. 1 and Table 2). 


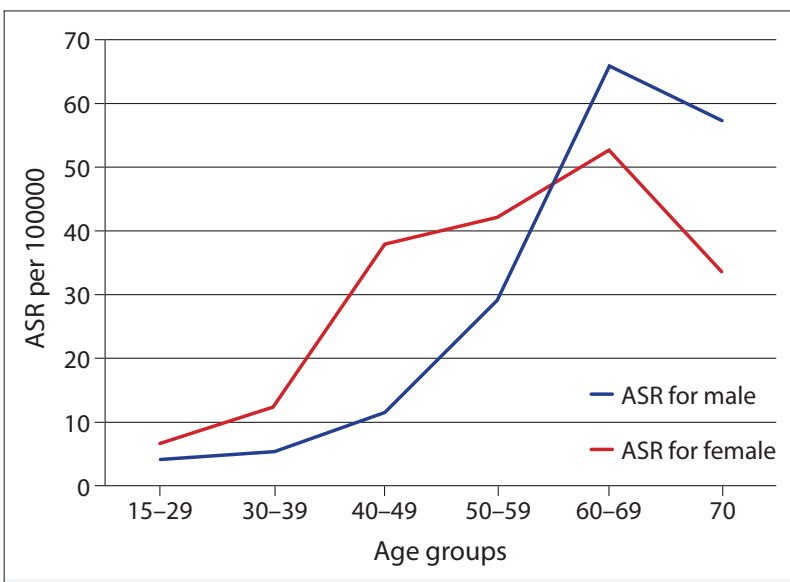

Fig. 1. ASR of cancers according to sex and age groups, North Cyprus; 2010-2014.

As can be seen in Table 3, the crude incidence rates for adult male and female cancer patients for the years between 2010-2014 were 149.81 and 187.24 per 100,000 North Cyprus resident population in the 15 or over age group per year, respectively. The rate for women was significantly higher than for men, which means that this difference was statistically significant $(p<0.001)$. The corresponding age standardized incidence rates (ASRs) were 180.61 and 192.75 per 100,000 person-years. Analysis of the rates in 2010, 2011, 2012 and 2014 revealed that incidence rates for women were higher than for men and this difference was found to be statistically significant in 2010, 2011 and 2012 $(\mathrm{p}<0.001$ for each year) but not statistically significant in $2014(\mathrm{p}=0.089)$. The rate for men in 2013 was greater than for women; however, this difference was not statistically significant $(\mathrm{p}=0.8930)$.

The crude incidence rates for both male and female cancer patients excluding non-melanoma skin cancers for the years between 2010-2014 were 143.91 and 179.89 per 100,000 North Cyprus resident population in the 15 or over age group per year, respectively. The corresponding age-standardized incidence rates were 173.7 and 185.52 per 100,000 person-years.

Selected Cancers and Comparison with Globocan 2012 Data: Comparisons between NC and SE and between NC and SC should be interpreted with caution (Fig. 2 and Fig. 3). Further details on prevalent cancers during the study period (2010-2014) are as follows:

All cancers excluding non-melanoma skin cancers: The mean age of the patients was 60.4 (CI 95\%: 59.761.1). The mean age of male patients was 63.3 and the mean age of female patients was 58.8. The average age difference between men and women was statistically different $(\mathrm{p}<0.001)$. In total, $47.1 \%$ of the patients were male and $52.9 \%$ of them were female. The ASR in NC for adult women and men was significantly lower than in SC and SE.

Breast Cancer (C50): During the study period, only 12 adult men with breast cancer were recognized, therefore breast cancer was investigated only for women. The mean age of the patients was 58.7 years (CI 95\%: 57.3-60.0). The ASR in adult women in NC was significantly lower than the ASR in SC and SE.

Thyroid Cancer (C73): The mean age of the thyroid cancer patients was 48.1 (CI 95\%: 46.2-49.9). The mean age between the two genders was not different as the mean age for males was 48.5 and for females was 47.9 ( $\mathrm{p}=0.7868) .24 .5 \%$ of the patients with thyroid cancer were male and $75.5 \%$ of them were female. The ASR in adult women in NC was statistically the same as the ASR for SC's adult women ( $\mathrm{p}=0.0031)$; however, it was significantly greater than the ASR in SE's adult women. For men, the ASR in NC was statistically the same as the ASR in both SC and SE's adult men ( $\mathrm{p}=0.0668$, $\mathrm{p}=0.1112$ ).

Colorectal Cancer (C18-21):The mean age of the patients with colorectal cancer was 65.8 (CI 95\%: 63.967.7). The mean age between the two genders was not

Table 3 Crude Incidence Rate (CIR) and Age Standardized Rate (ASR) of cancers, per 100000 population aged 15 and over, according to gender and year, North Cyprus

\begin{tabular}{|c|c|c|c|c|c|c|c|c|}
\hline \multirow[t]{2}{*}{ Year } & \multicolumn{4}{|c|}{ Male } & \multicolumn{4}{|c|}{ Female } \\
\hline & $\mathbf{n}$ & $\%$ & CIR & ASR (CI 95\%) & $\mathbf{n}$ & $\%$ & CIR & ASR (CI 95\%) \\
\hline 2010 & 92 & 11 & 82.13 & $99.63(83.84-115.42)$ & 135 & 14.3 & 134.02 & 140.35 (121.84-158.86) \\
\hline 2011 & 158 & 18.8 & 141.06 & $171.02(150.33-191.71)$ & 185 & 19.6 & 183.66 & $188.56(167.29-209.82)$ \\
\hline 2012 & 123 & 14.7 & 109.81 & $131.01(113.01-149.02)$ & 172 & 18.2 & 170.76 & 175.35 (154.82-195.87) \\
\hline 2013 & 241 & 28.7 & 215.16 & $260.98(235.44-286.52)$ & 214 & 22.7 & 212.45 & 218.83 (195.90-241.77) \\
\hline 2014 & 225 & 26.8 & 200.87 & $240.69(216.25-265.12)$ & 237 & 25.1 & 235.29 & 240.67 (216.69-264.64) \\
\hline Total (2010-2014) & 839 & 100 & 149.81 & 180.67 (171.17-190.16) & 943 & 100 & 187.24 & 192.75 (183.12-202.38) \\
\hline
\end{tabular}




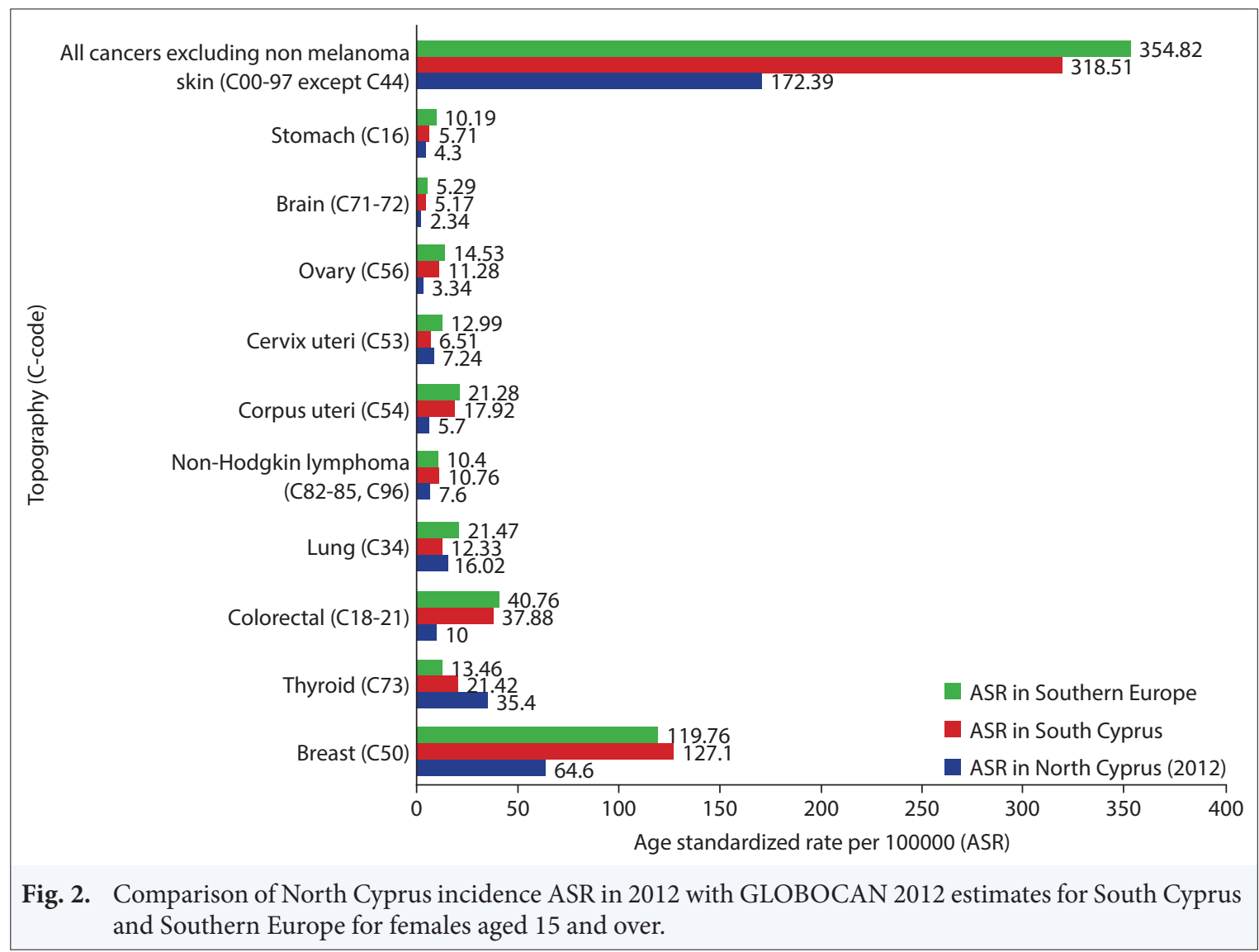

statistically different as the mean age for males was 65.5 and for females was 66.4 ( $\mathrm{p}=0.6587)$. A total of $65.5 \%$ of the patients were male and $34.5 \%$ of them were female. For women, the ASR in NC was significantly lower than the ASR in SC and SE. However, for men, the ASR in NC was statistically lower than the ASR in SE but statistically the same as in SC ( $\mathrm{p}=0.0188)$.

Lung Cancer (C34):The mean age of the patients with lung cancer was 65.1 (CI 95\%: 63.6-66.6). The mean age between the two genders was statistically different. The mean age of the male patients was 66.7 and the mean age of the female patients was 58.7. A total of $80.5 \%$ of the patients were male and $19.5 \%$ of them were female. For women, the ASR in NC was statistically the same as in both SC and SE ( $\mathrm{p}=0.8577$, $\mathrm{p}=0.0465)$. In the same manner, for men, the ASR in NC was statistically the same as in both SC and SE ( $\mathrm{p}=0.9878, \mathrm{p}=0.002)$.

Non-melanoma skin (C44): The mean age of the patients with non-melanoma skin cancer was 67.1 (CI 95\%: 64.2-70.0). The mean age between the two genders was not statistically different $(p=0.3644)$. The mean age of the male patients was 68.5 and the mean age of the female patients was 65.8 . A total of $52.9 \%$ of the patients were male and $47.1 \%$ of them were female.

Stomach (C16): The mean age of the patients with stomach cancer was 65.5 (CI 95\%: 62.4-68.6). The mean age between the two genders was not statistically different $(\mathrm{p}=0.1428)$. The mean age of the male patients was 63.4 and the mean age of the female patients was 68.1 . A total of $55.1 \%$ of the patients were male and $44.9 \%$ of them were female. For women and men in NC, the ASR was statistically the same as in SC $(p=0.2266, p=0.0475)$ but significantly lower than in SE.

Non-Hodgkin Lymphoma (C82-85, C96): The mean age of the patients was 58.1 (CI 95\%: 54.1-61.9). The mean age of the two genders was not statistically different $(\mathrm{p}=0.7370)$. The mean age of the male patients was 57.4 and the mean age of the female patients was 58.7 . A total of $52.1 \%$ of the patients were male and $47.9 \%$ of them were female. For women in NC, SC and SE, the ASR values were statistically the same $(\mathrm{p}=0.0853, \mathrm{p}=0.0778)$. In Addition, for men in NC, $\mathrm{SC}$ and SE, the ASR values were statistically the same $(\mathrm{p}=0.2327, \mathrm{p}=0.0197)$. 


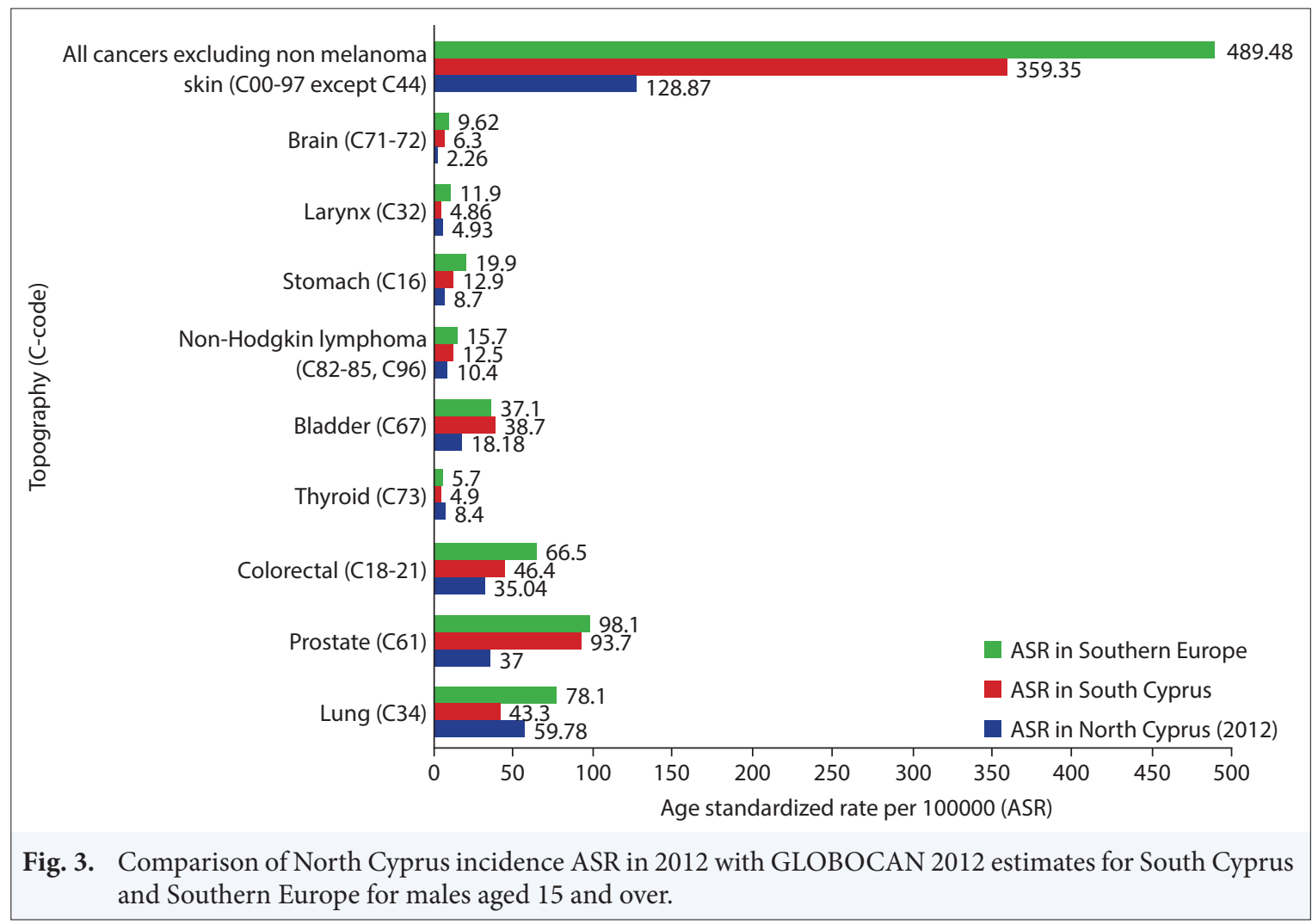

Bladder (C67): $74.1 \%$ of the bladder cancer patients were male and the mean age of those male patients was 68.15 (CI 95\%: 64.03-72.27). The ASR value in NC for adult men was significantly lower than in SC and SE.

Cervix uteri (C53): The mean age of the patients was 57.0 (CI 95\%: 53.1-62.3). The ASR in NC for adult women was significantly lower than in SE. However, it was statistically the same as SC's ASR.

Corpus uteri (endometrium) (C54): The mean age of the patients was 69.6 (CI 95\%: 66.1-73.0). The ASR value in NC for adult women was significantly lower than the ASR in SC and SE.

Ovarian (C56): The mean age of the patients who had ovarian cancer was 59.3 (CI 95\%: 54.5-64.1). The ASR value in NC was significantly lower than the ASR in SC and SE.

Prostate (C61): The mean age of the prostate cancer patients was 69.9 (CI 95\%: 68.1-71.3). The ASR value in $\mathrm{NC}$ for men was significantly lower than in $\mathrm{SC}$ and SE.

Brain (C71-72): The mean age of the patients who had brain cancer was 48.5 (CI 95\%: 43.5-53.6). The mean age between the two genders was statistically different. The mean age of the male patients was 67.4 and the mean age for female patients was 44.4. A total of
$52.3 \%$ of the patients were female and $47.7 \%$ of them were male. For men, the ASR in NC was statistically the same as in SC $(p=0.0044)$ but was statistically lower than in SE. For women, the ASRs in NC, SC and SE were statistically the same $(\mathrm{p}=0.0387, \mathrm{p}=0.0107)$.

Larynx (C32): From 2010 to 2014, no cases of larynx cancer was observed in females; however, 30 male patients were observed. The mean age of male patients was 65.4 (CI 95\%: 62.7-68.2). In addition, the ASRs for female patients in SE and SC were lower with 0.98 and 0.58 (almost zero), respectively. For male patients, the ASRs in NC and SC were statistically the same $(p=0.5120)$ and the ASR in NC was significantly lower than in SE.

According to a recent study concerning the incidence of cancer in Turkey (2009), the most prevalent cancers in women were breast, thyroid and colorectal cancers. In men, lung, prostate and bladder cancers were more common.[12]

2010-2014 head and neck, upper gastrointestinal, hematological and gynaecological cancers were compared separately, as seen in Table 1:

The most frequent head and neck cancers (83 total cases, $4.7 \%$ of all cancers cases) were firstly lip and oral cavity (33 cases, $39.8 \%$ of head and neck cancers), 
secondly male larynx (30 cases, $36.1 \%$ ) and lastly nasopharnyx (11 cases, 13.3\%). A similar ranking was obtained for only males with larynx (30 cases, $46.2 \%$ ), liporal cavity (22 cases, $33.8 \%$ ) and nasopharynx ( 7 cases, $10.8 \%$ ) were observed. No cases of larynx cancer were observed in female patients but lip-oral cavity cancers (11 cases, 61.1\%) were most commonly observed.

As colorectal cancers were evaluated separately, the most frequent upper gastrointestinal cancers (129 total cases, $7.2 \%$ of all cancer cases) were stomach (51 cases, $39.6 \%$ of upper GI cancers), pancreas (28 cases, $21.7 \%$ ) and liver (27 cases, $20.9 \%$ ). In male patients, the rankings were found to be stomach (27 cases, $37.5 \%$ ), liver (17 cases, $23.6 \%$ ) and pancreas (17 cases, $23.6 \%$ ), respectively. The same rankings were found for female patients as were found in the overall statistics with stomach (24 cases, $42.1 \%$ ), pancreas (11 cases, $19.3 \%$ ) and liver (10 cases, $17.5 \%)$.

The most frequent hematological cancers (136 cases, $7.6 \%$ ) in total were non-Hodgkin ( 73 cases, $53.7 \%$ of hematological cancers), Hodgkin (28 cases, $20.6 \%$ ) and multiple myloma (26 cases, 19.1). The same ranking was observed in females with non-Hodgkin (35 cases, 53.0\%), Hodgkin (15 cases, 22.7\%) and multiple myeloma (11 cases, 16.7\%). However, the male patient ranking was non-Hodgkin (38 cases, 54.3\%), multiple myeloma (15 cases, $18.6 \%)$ and Hodgkin (13 cases, $18.6 \%)$.

In gynaecological cancers, corpus uteri (29 cases, $33.3 \%$ ), ovarian ( 27 cases, $30.7 \%$ ) and cervix cancers ( 24 cases, $27.2 \%)$ were observed most frequently. As seen from these results, there were no statistically differences between the incidences of gynaecological cancers.

\section{Discussion}

NEU Hospital was founded in 2010. Today, NEU Hospital is the only actively operating university hospital in Northern Cyprus. NEU Hospital was the only hospital that has a radiation oncology department until July 2016 and PET/CT equipment tii now. Because of these facilities, patients with oncology diagnosis requiring treatment are admitted to the hospital for further evaluation and/or treatment. The diagnosis and treatment of other patients who are not admitted are carried out overseas. Therefore, one can say that the results obtained demonstrate that NEU Hospital represents a significant portion of the population in NC, but not its entirety. As a result, it should be stipulated that this research is not a countrywide Cancer Registry study. Between the years 2010 and 2014, increases or decreases in the number of patients according to the types of cancer are not related to the overall incidence, but are related to the changing numbers of patients admitted to the hospital. Accordingly, we cannot comment accurately on the annual increase or decrease in the number of patients. For example, from 2010 to 2014 , there was a statistical increase in prostate cancer at NEU Hospital. The significant reason for this is that the role of radiotherapy in treating prostate cancer is particularly important and the increase in the number of patients admitted was related to the introduction of the latest technology in radiotherapy equipment that ensures more effective treatment. As NEU Hospital does not have a pediatric oncology clinic, our research does not therefore include patients under 15 years old.

According to the results obtained from case between the years 2010-2014, the most common cancers in males were lung, prostate, colorectal, thyroid and bladder cancer. For females, the following cancers were most frequently observed: breast, thyroid, colorectal, lung and non-melanoma skin cancer. According to the studies, both red meat/high-fat diets and smoking were identified as factors that could increase the risk of colorectal cancer.[13,14,15] High-fat diets increase cholesterol and bile acid metabolites in the colon. In tests on laboratory animals, these metabolites increased cell proliferation, accelerated tissue growth and led to the development of colon cancer. The main reason why colorectal cancer is frequently observed in males and females in Northern Cyprus is due to the fact the consumption culture of Turkish Cypriot people is widely considered to involve increased meat eating and smoking. This type of cancer is defined as the third most common cancer in the world. [14] The results obtained from NEU Hospital were consistent with the overall figures as, according to our results, colorectal cancer in males and females was observed as the third most prevalent cancer.

According to the results, the most common cancer out of upper gastrointestinal cancers in males and females is stomach cancer. Due to the consistently good weather conditions, Turkish Cypriot people can frequently be found grilling meat on the barbecue; it is thought that frequent consumption of grilled meat and the recurrent use of burnt oils may cause stomach cancer. Previous studies have indicated that high consumption of red meat (especially barbecue) increases the risk of stomach cancer.[13] In addition, nitrates and nitrites that are especially found in salted and smoked meat products (salami, sausage and pickled foods etc.) are available in large quantities and carry carcinogenic 
characteristics to the stomach.[16] Turkish Cypriots use these products frequently and there is a particularly high consumption of a popular salami called "bolibif", which may also increase the risk of stomach cancer.

In 2012, breast cancer was defined as the most prevalent disease among women with an estimated 1.67 million new cancer cases worldwide; it represents about $25 \%$ of all cancers.[17] Breast cancer is one of the cancer types that occurs most frequently in females. It occurs globally, in developed countries as well as in developing countries.[17] In our research, breast cancer was considered only for females, because there were very few males (only 12) who had breast cancer in the results. The results obtained demonstrate that breast cancer is the most prevalent form of cancer in females in NC, as it is globally. The main known breast cancer risks that cannot be changed are age, gender, early menarche, family history and late menopause. $[18,19,20]$ Nevertheless, additional aspects are related with increased breast cancer risk, such as the use of both estrogen and progestin menopausal hormones, cigarette smoking, lack of physical activity, alcohol consumption, being overweight and obesity, and they are all changeable. [21,22] It is thought that the reason why breast cancer in females in Northern Cyprus is considered to be high risk is that in Mediterranean countries, females generally experience late menopause and early menstruation.[20] In addition, the profile of Turkish Cypriots females is typically that they are overweight, well fed and most of them have inactive and passive life styles. In this respect, the possibility of contracting breast cancer in Northern Cyprus is high and this could be considered to be because of inactivity, being overweight and prevalent obesity.

Lung cancer was the most frequently identified cancer and a significant cause of global cancer deaths among males, especially in less developed countries. [15] Among females, lung cancer ranked third and was the second leading cause of cancer death.[15] In addition, according to the Globocan results that were published in 2012, lung cancer in males occurred twice as much than in females.[11] Our results are in line with the overall figures in this regard. According to the results observed, males develop lung cancer more than females; it is the most common cancer in males and the fourth most common cancer in females. One of the main risk factors is smoking, for both males and females. The relationship of lung cancer with smoking was first demonstrated in 1950 and was confirmed by subsequent studies.[23] According to the results of studies on the risk of lung cancer, the incidence was found to be 24-36 times higher in smokers. For $90 \%$ of lung cancer cases diagnosed in males and in $80 \%$ of female cases, the cause of the disease was considered to be smoking.[24] Internationally, approximately $40 \%$ of the world's male population are smokers compared with approximately $9 \%$ female smokers. In North Cyprus, lung cancer was the most frequent occurring cancer in males and ranked fifth for females. This is due to the high rate of tobacco use in Northern Cyprus by both genders.

Furthermore, among head and neck cancers in males, the most common type of cancer observed was larynx cancer, while bladder cancer was also prevalent in the male population. The major risk factor for bladder and larynx cancer is smoking cigarettes.[25,26] Along with lung cancer, the rates of incidence for these types of cancers is found to be statistically higher for men than for women, both globally and in Northern Cyprus.[11] In fact, the research carried out for the years 2010-2014 revealed that larynx cancer was not observed in any women. All these results can be considered to be because men consume more cigarettes compared to women. In 2008, according to a survey that was performed in Northern Cyprus on drug use among adults, the proportion of men who smoked cigarettes at some point in their lives was $73.7 \%$ and for woman it was found to be $57.3 \%$. The proportion of men who used cigarettes more intensively was $60.1 \%$ and for women it was $37.3 \%$, a rate that is approximately two times greater.[27] In this study, it is supported that, in Northern Cyprus, males consume more cigarettes than females.

According to the results obtained for the years of 2010-2014, thyroid cancer ranked second in women and fourth in men and therefore was one of the most frequent types of cancer. The frequency varied according to age and gender. It was observed infemales $(75.5 \%)$ more than in males $(24.5 \%)$. Thyroid cancer mostly occurred among people aged $45-54$ and is seen most commonly in women.[28] According to our research, the average age is concurrent with the overall statistics as the average age for men was 49 and for women was 48. Differentiated thyroid cancer (papillary and follicular) originate from follicular cells. In our hospital, $90 \%$ of cases were papillary thyroid cancer and $10 \%$ were follicular. Exposure to radiation is a proven risk factor for thyroid cancer; however, there have been no known nuclear accidents that have occurred in or around North Cyprus. As thyroid cancer on the island has a high risk and the genetic pool is narrow, this kind of cancer may frequently be observed, 
depending on the mutation. Hashimoto's thyroiditis is the main reason for hypothyroidism and it is also common in females on the island. The lymphocytic infiltration of Hashimoto's thyroiditis is normally connected with papillary thyroid cancer and may be a risk factor for producing this type of cancer.[29,30] It was thought that thyroid cancer is caused by iodine deficiency in a study conducted in Northern Cyprus.[31] However, there is a need for etiopathogenetic study results to determine the exact cause or causes. Moreover, research carried out on the incidence of cancer in Northern Cyprus between the years 1990-2004, revealed that thyroid cancer was not even in the top ten most prevalent cancers in both males and females.[32] The reason for this could be due to the lack of developed methods used in the diagnosis and treatment of thyroid cancer and the lack of advanced devices in the nuclear medicine department at that time in Northern Cyprus. Because of this, all patients were required to travel abroad for diagnosis and treatment.

Among the most common gynecologic cancers, endometrium (corpus uteri) cancer also contains similar etiologic factors as breast cancer. The reason why endometrial cancer has increased in females is due to an increase in being overweight, suffering from obesity and a general lack of physical activity. $[33,34]$ As mentioned before regarding the breast cancer risk factors in Northern Cyprus, endometrial cancer in females is also common among gynecologic cancers. The incidence of cervix cancer in NC was seen in almost half of the incidence in SE. As North Cyprus is an island and has a smaller population, it is easier to control sexually transmitted diseases, which may cause HPV (human papilloma virus) infection of the cervix. [35]

Non-melanoma skin cancer was observed in females in fifth place and in males, it ranked in seventh place. Studies have indicated increased sun-exposure is a high risk factor for non-melanoma skin cancers. It is believed that because our island experiences almost 12 months of sun exposure, the risk of skin cancer is frequently observed.

According to the statistics stated in both Northern and Southern Cyprus, there is no significant statistical difference between both the ASR values in females with thyroid, lung, stomach, NHL, brain, cervix, and larynx cancers and in males with thyroid, lung, colorectal, stomach, NHL, larynx, and brain cancers. Based on these results, we can specify that Northern and Southern Cyprus both have similar environmental factors and culture of life. In 2009, according to the Turkish Cancer Statistics, the most common female cancers were breast, thyroid and colorectal cancers; the most frequent male cancers were lung, prostate and bladder cancers.[12] These results are very similar to our results in Cyprus. The reason for these results could be the similar life styles of both populations and ongoing migration between the two countries.

Overall, the mean age of female patients was significantly lower than the mean age of men. An explanation for this is that the cancer most experienced by males was prostate cancer in older ages (average age: 70). Furthermore, females most commonly experienced thyroid cancer in middle ages (average age: 48 ). This result is also consistent with the global statistics that show that prostate cancer is the most common malignancy among elderly men and thyroid cancer is most frequently diagnosed among people aged 45-54.[28,36]

As one can see, most common cancer risk factors are due to the consumption of cigarettes, poor eating habits and the lack of physical activity.[37,38] This is very important because these factors can be changed. Consequently, it is firstly recommend that the authorities should prepare and implement programs for smoking prevention.[27] Secondly, people should increase their levels of physical activity. This can be achieved by establishing programs that inform individuals and communities how to be active, and by developing a policy to motivate and support them, which may contribute the prevention of cancer. In addition, the state authorities and organizations should plan and inform the community as a whole about proper nutrition, the risks of obesity and should encourage an increase in physical activities.

We have discussed the possible risk factors of the most common cancers in NC, however, there is a need for etiopathogenetic scientific results. This means that risk factor studies can be carried out to inform the public and to contribute significantly to future preventative measures. Additionally, the development of cancer registry centers,switching to an active cancer registry system and combining the data obtained from all health care providers under a single cancer organisation are recommendedin order to obtain more comprehensive data.

\section{Acknowledgments}

This research is the project of NEU Hospital. We hereby thank all the doctors and staff of the Radiation Oncology Department, Nuclear Medicine Department and all other departments in the hospital for their assistance and support during the investigation, including primarily the radiation oncology department. Also, we would 
like to individually thank Prof. Dr. Suna Kıraç, Asst. Assoc. Dr. Deniz Bedel in Nuclear Medicine, Assoc. Dr. Orgun Deren in Plastic Reconstructive and Aesthetics Surgery, Dr. Didem Mullaaziz in Dermatology and Prof. Dr. Ali Ulvi Önder in the Urology Department. We also wish to thank Mr. Simon Thompson for language editing and proofreading of the manuscript.

\section{Disclosure Statement}

The authors declare no conflicts of interest.

\section{References}

1. Parkin DM, Whelan SL, Ferlay J, Gao YT, Ferlay J, Powell J. Cancer Incidence in Five Continents, Vol. VI. IARC Scientific Publications, No. 120: Lyon, IARC; 1992.

2. Parkin DM, Whelan SL, Ferlay J, Raymond L, Young J. Cancer Incidence in Five Continents, Vol. VII. IARC Scientific Publications, No. 143: Lyon, IARC; 1997.

3. World Health Organization, International Agency for Research on Cancer. World cancer report 2014.

4. World Health Organization. Fact sheet $N^{\circ} 297$. Available at: http://www.who.int/mediacentre/factsheets/ fs297/en/. Accessed May 18, 2017.

5. Hocking B. Cancer in Korean War Navy technicians: mortality survey after 40 years. Am J Epidemiol 2003;157(3):279.

6. Gray GC, Smith TC, Kang HK, Knoke JD. Are Gulf War veterans suffering war-related illnesses? Federal and civilian hospitalizations examined, June 1991 to December 1994. Am J Epidemiol 2000;151(1):63-71.

7. World Health Organization. International statistical classification of diseases and related health problems. Vol. 1; 2014.

8. TRNC State Planning Organization. TURKISH REPUBLIC OF NORTHERN CYPRUS: The Organization; c2001-2015. Statistical Year Book 2011. Available at: http://www.devplan.org/ISTYILLIK/ISTYILLIK-2011.pdf. Accessed May 18, 2017.

9. Boyle P, Parkin DM. Cancer registration: principles and methods. Statistical methods for registries. IARC Sci Publ 1991;(95):126-58.

10. Doll R, Payne P, Waterhouse J. Cancer Incidence in Five Continents, Vol. I. A Technical Report 1966. Geneva, UICC; Berlin: Springer-Verlag.

11. Globocan 2012 France: International Agency for Research on Cancer. Available at: http://globocan.iarc.fr/ Default.aspx. Accessed May 18, 2017.

12. Gültekin M, Boztaş G. Türkiye Kanser İstatistikleri.
Sağlık Bakanlığı, Türkiye Halk Sağlığ Kurumu 2014;43. Available at: http://kanser.gov.tr/Dosya/ ca_istatistik/2009kanseraporu.pdf. Accessed May 18, 2017.

13. Hu J, La Vecchia C, Morrison H, Negri E, Mery L; Canadian Cancer Registries Epidemiology Research Group. Salt, processed meat and the risk of cancer. Eur J Cancer Prev 2011;20(2):132-9.

14. Haggar FA, Boushey RP. Colorectal cancer epidemiology: incidence, mortality, survival, and risk factors. Clin Colon Rectal Surg 2009;22(4):191-7.

15. Torre LA, Bray F, Siegel RL, Ferlay J, Lortet-Tieulent J, Jemal A. Global cancer statistics, 2012. CA Cancer J Clin 2015;65(2):87-108.

16. Lazarevic K, Nagorni A, Rancic N, Milutinovic S, Stosic L, Ilijev I. Dietary factors and gastric cancer risk: hospital-based case control study. J BUON 2010;15(1):89-93.

17. Globocan 2012. France: International Agency for Research on Cancer. Breast Cancer Estimated Incidence, Mortality and Prevalence Worldwide in 2012.

18. Alteri R, Barnes C, Burke A. Breast Cancer Facts \& Figures 2013-2014. Atlanta: American Cancer Society; 2013.

19. World Health Organization. Switzerland: WHO 2015 Breast Cancer.

20. Griffiths K, Wilson DW, Singh RB, De Meester F. Effect of dietary phytoestrogens on human growth regulation: imprinting in health \& disease. Indian J Med Res 2014;140 Suppl:S82-90.

21. Kushi LH, Byers T, Doyle C, Bandera EV, McCullough M, McTiernan A, et al; American Cancer Society 2006 Nutrition and Physical Activity Guidelines Advisory Committee. American Cancer Society Guidelines on Nutrition and Physical Activity for cancer prevention: reducing the risk of cancer with healthy food choices and physical activity. CA Cancer J Clin 2006;56(5):254-81.

22.van den Brandt PA, Spiegelman D, Yaun SS, Adami HO, Beeson L, Folsom AR, et al. Pooled analysis of prospective cohort studies on height, weight, and breast cancer risk. Am J Epidemiol 2000;152(6):51427.

23. Doll R, Hill AB. Smoking and carcinoma of the lung; preliminary report. Br Med J 1950;2(4682):739-48.

24. U.S. Department of Health and Human Services. The Health Consequences of Smoking: A Report of the Surgeon General. Atlanta: U.S.: 2004. Available at: https:// www.cdc.gov/tobacco/data_statistics/sgr/2004/complete_report/index.htm. Accessed May 18, 2017.

25. Cancer Research UK. Risks and causes of laryngeal cancer. 
26. Freedman ND, Silverman DT, Hollenbeck AR, Schatzkin A, Abnet CC. Association between smoking and risk of bladder cancer among men and women. JAMA 2011;306(7):737-45.

27. Çakıcı M, Çakıcı E, Karaaziz M, Tutar N, Eş A. Prevalence and Risk Factors of Drug Use in TRNC. Journal of Dependence 2014;15:159-66.

28. National Cancer Institute. United States: SEER Stat Fact Sheets: Thyroid Cancer. Surveillance, Epidemiology, End Results Program.

29. Lee JH, Kim Y, Choi JW, Kim YS. The association between papillary thyroid carcinoma and histologically proven Hashimoto's thyroiditis: a meta-analysis. Eur J Endocrinol 2013;168(3):343-9.

30. Noureldine SI, Tufano RP. Association of Hashimoto's thyroiditis and thyroid cancer. Curr Opin Oncol 2015;27(1):21-5.

31. Gökyiğit A, Demirdamar SR. Epidemiology and cost of drug treatment of cancer in Northern Cyprus. Turk J Med Sci 2016;46(3):769-74.

32. Hinçal E, Taneri B, Taneri U, Djamgoz MB. Cancer in- cidence in North Cyprus (1990-2004) relative to European rates. Asian Pac J Cancer Prev 2008;9(4):725-32.

33. John EM, Koo J, Horn-Ross PL. Lifetime physical activity and risk of endometrial cancer. Cancer Epidemiol Biomarkers Prev 2010;19(5):1276-83.

34. Kaaks R, Lukanova A, Kurzer MS. Obesity, endogenous hormones, and endometrial cancer risk: a synthetic review. Cancer Epidemiol Biomarkers Prev 2002;11(12):1531-43.

35. World Health Organization. Fact sheet $N^{\circ} 380$.

36. Stangelberger A, Waldert M, Djavan B. Prostate cancer in elderly men. Rev Urol 2008;10(2):111-9.

37. Thune I, Furberg AS. Physical activity and cancer risk: dose-response and cancer, all sites and site-specific. Med Sci Sports Exerc 2001;33(6 Suppl):S530-50.

38. Danaei G, Vander Hoorn S, Lopez AD, Murray CJ, Ezzati M; Comparative Risk Assessment collaborating group (Cancers). Causes of cancer in the world: comparative risk assessment of nine behavioural and environmental risk factors. Lancet 2005;366(9499):178493. 